\title{
Gap-Townes solitons and delocalizing transitions of multidimensional Bose-Einstein condensates in optical lattices
}

\author{
Mario Salerno $^{1}$, F. Kh. Abdullaev ${ }^{2}$, and B. B. Baizakov ${ }^{2}$ \\ ${ }^{1}$ Dipartimento di Fisica "E.R. Caianiello" and Consorzio Nazionale \\ Interuniversitario per le Scienze Fisiche della Materia (CNISM), Universitá di \\ Salerno, I-84081 Baronissi (SA), Italy \\ 2 Physical-Technical Institute of the Uzbek Academy of Sciences, \\ 100084, Tashkent, Uzbekistan
}

\begin{abstract}
We show the existence of gap-Townes solitons for the multidimensional Gross-Pitaeviskii equation with attractive interactions and in two- and three-dimensional optical lattices. In absence of the periodic potential the solution reduces to the known Townes solitons of the multi-dimensional nonlinear Schrödinger equation, sharing with these the propriety of being unstable against small norm (number of atoms) variations. We show that in the presence of the optical lattice the solution separates stable localized solutions (gap-solitons) from decaying ones, characterizing the delocalizing transition occurring in the multidimensional case. The link between these higher dimensional solutions and the ones of one dimensional nonlinear Schrödinger equation with higher order nonlinearities is also discussed.
\end{abstract}

\section{Introduction}

One interesting phenomenon occurring in ultracold atomic gases trapped in periodic potentials is the possibility to localize matter in states which can stay for a long time due to an interplay between nonlinearity, dispersion and periodicity. Such states (also called gap-solitons) have been observed in Bose-Einstein condensates (BEC) and in arrays of nonlinear optical waveguides 112 3/4. For attractive atomic interactions in BEC and in absence of a periodic potential, stable localized solutions are possible only in a one-dimensional (1D) setting since in two (2D) and three (3D) dimensions the phenomenon of collapse appears [5]. More precisely, one observes that when the number of atoms exceeds a critical threshold, the solution collapses in a finite time (blow-up) while for number of atoms below the critical threshold there is an irreversible decay of the state into background radiation. For the higher dimensional nonlinear Schödinger (NLS) equation, however, it is known that there exists an unstable localized solution, the so called Townes soliton [6], which separates decaying solutions from collapsing ones. Townes soliton, however, exists only for a single value of the number

\footnotetext{
^ E-mail: salerno@sa.infn.it
} 
of atoms, being unstable against fluctuations around it (for slightly overcritical or undercritical number of atoms the solution collapses or decays, respectively). The situation is drastically changed in presence of an optical lattice (OL). To this regard, it has been shown that stable $2 \mathrm{D}$ and $3 \mathrm{D}$ solitons can exists in OLs both in BEC and nonlinear optics contexts [7/8/9/10/11. Moreover, it is known that while the periodic potential can only marginally shift the critical value for collapse, it can substantially move the delocalizing transition curve, thereby increasing the soliton existence range in parameter space from a single point to a whole interval [12. The typical situation with 2D and 3D BEC solitons in OLs is therefore the following: in the parameter space the stable localized solutions are confined from above by the collapse curve and from below by the delocalizing transition curve, thus, in contrast with the one dimensional case where there are no limits for the existence of localized states, strict limitations for soliton existence appear in multidimensional cases. From this point of view it is clear that for possible experimental observation of multidimensional BEC solitons the parameter design becomes very important. Since the collapse curve is only marginally affected by the periodic potential, to enlarge existence ranges of solitons it is of interest to give a full characterization of the delocalizing curve in parameter space.

The aim of this paper is just devoted to this, i.e. we characterize $2 \mathrm{D}$ and 3D delocalizing curves of gap-solitons in terms of an unstable solution of the multidimensional Gross-Pitaeviskii equation (GPE), which we call gap-Townes soliton. This solution can be viewed as a separatrix (it separates gap soliton states from extended (Bloch) states) and reduces to the known Townes soliton when the strength of the OL goes to zero. Similar solutions were found also for the 1D NLS equation with higher order nonlinearities in [13], where they were called gap-Townes solitons, and in 14 where they were termed Townes solitons. Conditions for the occurrence of the delocalizing transition phenomenon of onedimensional localized modes of several nonlinear continuous periodic and discrete systems of the nonlinear Schrödinger type were also recently discussed in [15. For the periodic multidimensional GPE the delocalizing curve has been characterized in [12] as the critical threshold for the existence of one bound state in an effective potential. The characterization given here, however, is more general since it is valid also for 1D NLS with higher order nonlinearities. To this regard we remark that in absence of confining potential the 2D and 3D GPE behaves similarly to the 1D NLS with quintic and septic nonlinearities, respectively. The interplay between dimensionality and nonlinearity has been used to investigate collapse in lower dimensional NLS on the basis of pure dimensional arguments. In particular, the critical condition for collapse has been characterized as $D(n-$ 1) $-4=0$, where $n$ is the order of the nonlinearity in the equation and $D$ is the dimensionality of the system [16]. In the following we take advantage of this interplay to construct approximate gap-Townes soliton solutions of the GPE with multidimensional separable OLs, in terms of products of exact gap-Townes solutions of the 1D NLS with higher nonlinearities. Remarkably, we find that, except for strengths of the optical lattices very small, our approach produces very 
accurate gap-Townes solutions of multidimensional GPE with OL, thus giving an evident computational advantages. The results obtained in this paper can be seen as a generalization of the existence of gap-Townes solitons in the quintic NLS discussed in [13]14 to the case of the multidimensional Gross-Pitaeviskii equation.

We finally remark that the obtained results can also be applicable for photonic lattices with Kerr type of optical nonlinearity where the existence of a critical threshold for the lattice solitons has been observed [17.

The paper is organized as follows. In section II we introduce the model equations and discuss the link between multidimensional GPE with a separable trapping potential and the corresponding 1D NLS equation with higher order nonlinearity. We use a self consistent approach to approximate gap-Townes solitons of the GPE with products of exact gap-Townes soliton of the corresponding 1D NLS equation with higher order nonlinearity. In Section III we discuss the existence of localized solutions in the multidimensional GPE with OL by means by a variational approach and compare $2 \mathrm{D}$ and $3 \mathrm{D}$ results with those obtained from the VA applied to the quintic and septic NLS, respectively. In section IV we perform a numerical investigation of the existence (delocalizing) threshold for gap-Townes solitons of the 2D and 3D GPE. Finally, in the last section we briefly summarize our main results.

\section{Model equations and existence of gap-Townes solitons}

Let us consider the following Gross-Pitaevskii equation in d-dimensions $(d=$ $1,2,3$,$) as a model for a BEC in an optical lattice 12$

$$
i \psi_{t}+\nabla_{d}^{2} \psi+\varepsilon\left[\sum_{i=1}^{d} \cos \left(2 x_{i}\right)\right] \psi+\gamma|\psi|^{2} \psi=0,
$$

where $\nabla_{d}^{2}$ denotes the d-dimensional laplacian, $\sum_{i=1}^{d} \cos \left(2 x_{i}\right)$ denotes a square optical lattice with strength $\varepsilon, \gamma$ is the coefficient of nonlinearity, and $x_{i}=x, y, z$ for $i=1,2,3$, respectively. Here we will be mainly interested in cases $d=2$ and $d=3$. The existence of localized solutions of the multidimensional GPE with periodic potential and positive and negative nonlinearities (atomic scattering lengths), has been previously investigated both by variational analysis and by direct numerical simulations. In the following we concentrate on a topic which was not discussed in previous works, namely the existence of gap-Townes solitons in the multidimensional GPE and its link to the phenomenon of delocalizing transition. Due to the instability properties of these solutions it is difficult to find them without an analytical guide. To this regard we take advantage of the fact that the periodic potential is separable and in spite of the nonlinearity of the system we look for factorized stationary solutions of the form

$$
\psi\left(x_{1}, \ldots, x_{d}\right)=\prod_{i=1}^{d} \phi_{i}\left(x_{i}\right) e^{-i \mu t} .
$$


In $2 \mathrm{D}$ case $(d=2)$ the substitution of the factorized ansatz into Eq. (11) gives:

$$
\frac{\phi_{1_{x x}}}{\phi_{1}}+\frac{\phi_{2_{y y}}}{\phi_{2}}+\varepsilon[\cos (2 x)+\cos (2 y)]+\gamma\left|\phi_{1}\right|^{2}\left|\phi_{2}\right|^{2}=-\mu .
$$

This equation can also be written as

$$
\begin{aligned}
& \phi_{1_{x x}}+\varepsilon \cos (2 x) \phi_{1}+\frac{\gamma}{2}\left|\phi_{2}\right|^{2}\left|\phi_{1}\right|^{2} \phi_{1}=-\mu_{1} \phi_{1}, \\
& \phi_{2_{y y}}+\varepsilon \cos (2 y) \phi_{2}+\frac{\gamma}{2}\left|\phi_{1}\right|^{2}\left|\phi_{2}\right|^{2} \phi_{2}=-\mu_{2} \phi_{2},
\end{aligned}
$$

with $\mu_{1}=\mu_{2}=\mu / 2$. By assuming $\phi_{1}=\phi_{2} \equiv \phi$ and adopting a diagonal coordinate $x=y \equiv \xi$ we have that Eqs. (4) become equivalent to the following $1 \mathrm{D}$ eigenvalue problem

$$
\phi_{\xi \xi}+\varepsilon \cos (2 \xi) \phi+\frac{\gamma}{2}|\phi|^{4} \phi=-\frac{\mu}{2} \phi .
$$

From this we see that there is a link between the 2D cubic NLS and the 1D quintic NLS which implies a rescaling of parameters as: $\gamma \rightarrow \frac{\gamma}{2}, \mu \rightarrow \frac{\mu}{2}$.

The above equations can be easily extended to the 3D GPE with periodic potential. In this case Eq. (5) will be replaced by the following 1D NLS equation with septic nonlinearity

$$
\phi_{\xi \xi}+\varepsilon \cos (2 \xi) \phi+\frac{\gamma}{3}|\phi|^{6} \phi=-\frac{\mu}{3} \phi
$$

from which we see that in this case parameters must be rescaled according to: $\gamma \rightarrow \frac{\gamma}{3}, \mu \rightarrow \frac{\mu}{3}$.

It is appropriate to mention that a factorized solution of the form (2) with the components solutions of the nonlinear eigenvalue problem (5) cannot be an exact solution of the $2 \mathrm{D}$ or $3 \mathrm{D}$ GPE, since, due to the nonlinearity, the problem is obviously not exactly separable. On the other hand, by imposing the coincidence of the solutions along the diagonal axis may be a constraint for a reasonable approximate solutions of the $2 \mathrm{D}$ and $3 \mathrm{D}$ problems, especially when the nonlinearity is small. In analogy with the 1D NLS with quintic nonlinearity investigated in Ref. [13] we expect that the delocalizing curve coincides with the existence curve of gap-Townes solitons for which it was shown that the critical number of atoms decreases with increasing the strength of the OL. This means that in a deep OL the effective nonlinearity required for the existence of a gap-Townes soliton is smaller and the problem may become effectively close to separable.

To check the correctness of this argument we construct factorized solutions (2) of the 2D GPE by means of a self-consistent method which allows to solve the 1D quintic NLS eigenvalue problem exactly (similar results can be obtained for the $3 \mathrm{D}$ case).

In the top panel of Fig. 1 we show a 2D gap-Townes soliton obtained from the product ansatz using exact (self-consistent) gap-Townes solitons of the corresponding quintic NLS equation in Eq. (5). The lower left and right panels 


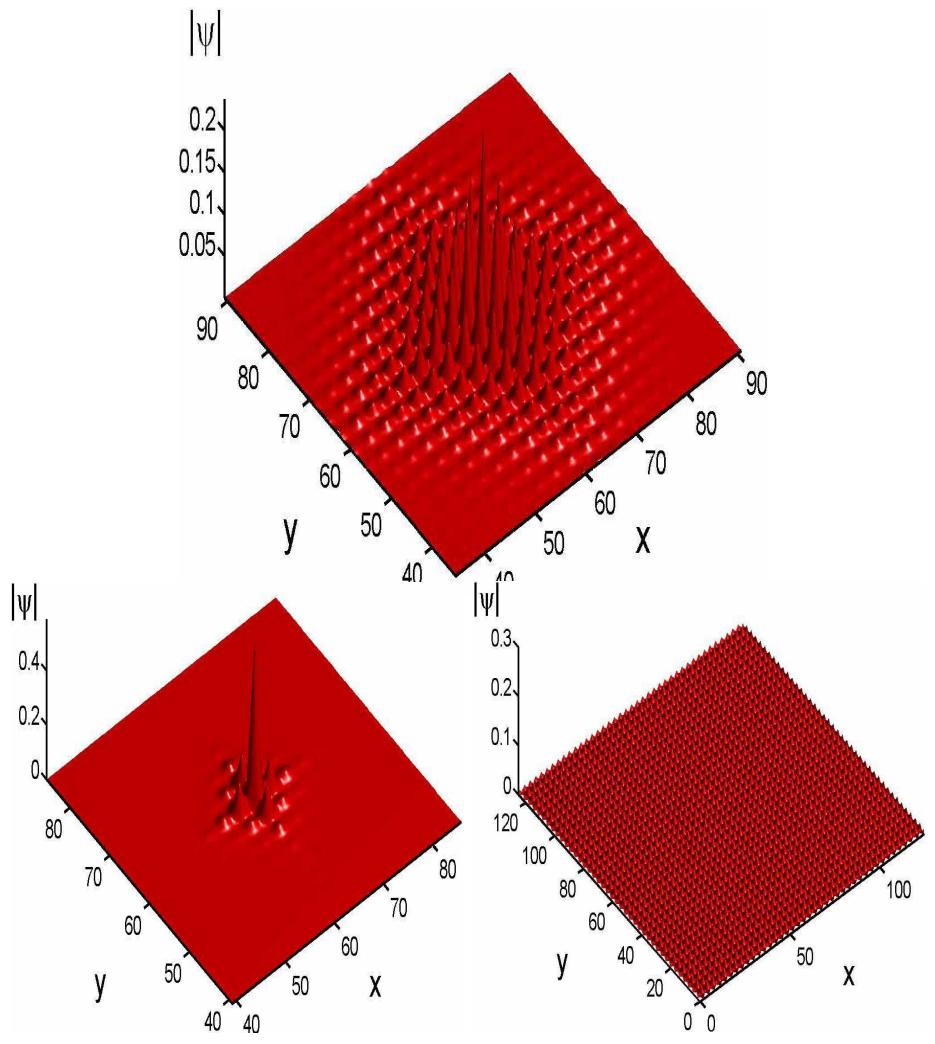

Fig. 1. Gap-Townes soliton (top panel), gap soliton (lower left panel) and Bloch state (lower right panel) of the GPE for parameter values $\varepsilon=5, \gamma=1$. The critical number of atoms in normalized units for the gap-Townes soliton is $N_{c}=0.4261$. The gap soliton and the Bloch state in the lower panel are obtained for slightly overcritical $(\mathrm{N}=0.4347)$ and undercritical $(\mathrm{N}=0.4176)$ values, respectively. 
show, respectively, the gap soliton and the extended Bloch state found at energy slightly below and slightly above (bottom of the lowest band) the one of the gap-Townes soliton. To check the reliability of the factorized ansatz we have computed the time evolution under the original 2D GPE equation using the factorized solution as initial condition. This is shown in Fig. 2 where the time evolution of the gap-Townes soliton in Fig. 1 (central panel) and the ones obtained for slightly overcritical and undercritical numbers of atoms are shown. We

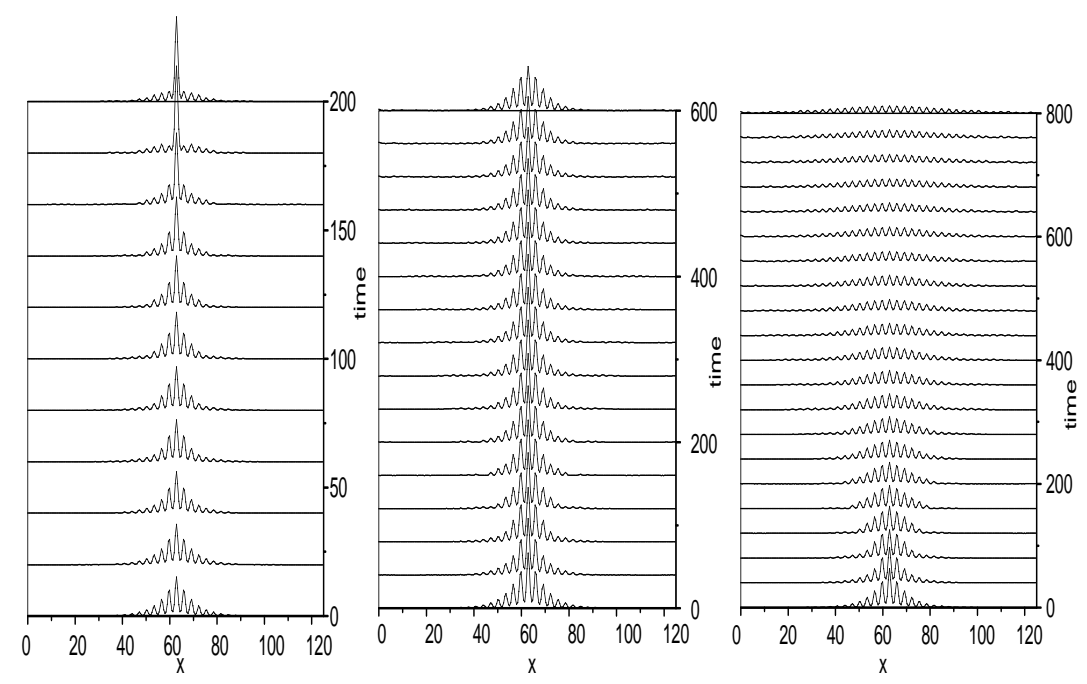

Fig. 2. Time evolution of the gap-Townes soliton shown in Fig. 1 with critical number of atoms (central panel) and for slightly overcritical (left panel) and undercritical (right panel) number of atoms.

see that while the product solution constructed from the quintic NLS remains localized for a long time, a slight increase or decrease of the number of atoms produces shrinking or decay of the solution, respectively. This clearly shows the existence and role of gap-Townes solitons of the 2D GPE with optical lattice in characterizing the delocalizing threshold.

\section{Variational analysis and existence of localized states}

The existence of localized states in multidimensional GPE with OL and in 1D NLS with higher order nonlinearities can be investigated by means of the variational approach (VA). To this regard we first consider Eq. (1) and search for stationary solutions of the form $\psi=U e^{-i \mu t}$ and consider the Lagrangian density 
associated with the equation for the stationary field $U$

$$
\mathcal{L}=\frac{1}{2}\left(\nabla_{d} U\right)^{2}-\frac{\mu}{2} U^{2}-\frac{\varepsilon}{2}\left[\sum_{i=1}^{d} \cos \left(2 x_{i}\right)\right] U^{2}-\frac{\gamma}{4} U^{4} .
$$

By taking a Gaussian ansatz for $U$

$$
U=A e^{-\frac{a}{2} \sum_{i=1}^{d} x_{i}^{2}}
$$

and performing spatial integration we obtain the following effective lagrangian $L_{e f f}=\int \mathcal{L} d x$ for parameters $A, a$

$$
L_{e f f}=\frac{A^{2}}{2}\left(\frac{\pi}{a}\right)^{\frac{d}{2}}\left[\frac{d}{2} a-\mu-d \varepsilon e^{-1 / a}-\frac{A^{2} \gamma}{2^{\frac{d}{2}+1}}\right], \quad d=1,2,3 .
$$

Variational parameters $A, a$, indicating the amplitude and inverse width of the localized state, are linked to the number of atoms by the relation $N=A^{2}\left(\frac{\pi}{a}\right)^{d / 2}$. From the conditions of stationarity of the effective lagrangian $\partial L_{e f f} / \partial a=$ $\partial L_{\text {eff }} / \partial A=0$, we get the following equations relating $N$ with $a$ and the chemical potential $\mu$

$$
\begin{aligned}
\mu & =\frac{d}{2} a-d \varepsilon e^{-1 / a}-\frac{\gamma N}{2^{d / 2}}\left(\frac{a}{\pi}\right)^{d / 2}, \\
N & =\frac{4 \pi^{d / 2}}{\gamma}\left(\frac{2}{a}\right)^{\frac{d}{2}-1}\left(1-\frac{2 \varepsilon}{a^{2}} e^{-1 / a}\right), \quad d=1,2,3 .
\end{aligned}
$$

In Fig. 3 we depict the $(N, \mu)$ curves obtained from the above transcendental equations for fixed values of $\varepsilon$ and for the cases $d=1,2,3$. We see that for the $1 \mathrm{D}$ case $d N / d \mu$ is always negative, this means, according to the Vakhitov-Kolokolov $(\mathrm{V}-\mathrm{K})$ criterion [18, that the solution exists and is stable for any value of $\varepsilon$ without limitations on the effective nonlinearity $N \gamma$. In the $2 \mathrm{D}$ case, a threshold in $N \gamma$ appears which is predicted by VA to be exactly $4 \pi$ for $\varepsilon=0$. Also notice that in this case $d N / d \mu<0$ is still satisfied for most branch curves, meaning that the solution is usually stable. The situation is quite different in the $3 \mathrm{D}$ case, where there is no limiting threshold for existence but most of the curves display a positive slope meaning that the solution is unstable. In particular, from the upper inset of the right panel we see that for curves close to the $\mu=0$ axis, $d N / d \mu$ change signs at $N \gamma \approx 70$ and becomes positive for higher values of $N \gamma$. The almost horizontal curves for lower values of $N \gamma$ are displayed in the lower inset of the figure, from which we see that $d N / d \mu$ changes from negative to positive after the curves have reached a maximum at values of $\mu$ which depend on $\varepsilon$.

Notice that for the $3 \mathrm{D}$ case the above equations predict for $\varepsilon=0$ the number of atoms dependence on the chemical potential as

$$
N=\frac{4 \pi \sqrt{\pi}}{\gamma \sqrt{-\mu}}
$$



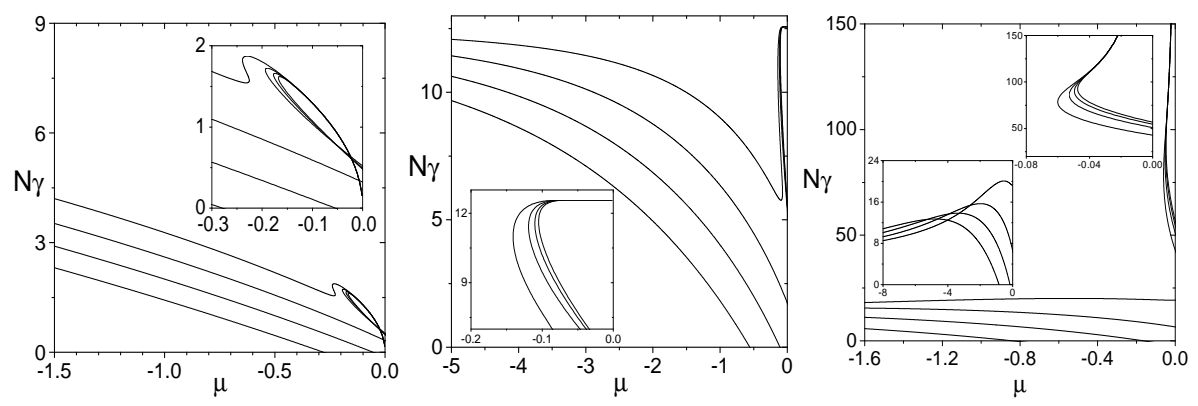

Fig. 3. Left panel. VA existence curves for the $1 \mathrm{D}$ case $(d=1$ in Eqs. (15) $)$ for different values of $\varepsilon$. The inset shows an enlargement for small values of $\mu$. Curves refer to $\varepsilon$ values increased in steps of 0.5 starting from $\varepsilon=0.5$ (upper curve and most left curve in the inset) to $\varepsilon=2.0$ (lower curve and most right curve in the inset). Central panel. Same as for the left panel but for the 2D case $(d=2$ in Eqs. (15)). The inset shows an enlargement for small values of $\mu$ close to the threshold $N=4 \pi$. Right panel. Same as for the left panel but for the 3D case $(d=3$ in Eqs. (15)). The upper inset shows an enlargement of the curves close to the $\mu=0$ axis, while the lower inset displays the curves close to the $N \gamma=0$ axis, on a larger scale.

Similar dependence $N$ vs. $\mu$ was previously obtained for stationary solutions of 3D NLS with cubic and quintic nonlinearity [19. Also notice that according to $\mathrm{V}-\mathrm{K}$ criterion this solution is unstable. From the condition $N \geq 0$, we obtain the limitation on the soliton width as

$$
\frac{2 \varepsilon}{a^{2}} e^{-1 / a} \leq 1,
$$

while for $\varepsilon>>1$ we obtain that the soliton exists if the width satisfies the condition

$$
a<a_{c 1} \approx \frac{1}{2(\log (\delta)+\log (\log (\delta)))}, \delta=2 \sqrt{2 \varepsilon},
$$

and $a>a_{c 2} \approx \sqrt{2 \varepsilon}-1 / 2$. For $\varepsilon=5$, for example, we obtain $a_{c 1}=0.2$, while the exact value is $a_{e} \approx 0.17$.

In view of the analogy of the 2D and 3D GPE with the 1D NLS with quintic and septic nonlinearity, respectively, it is of interest to compare the above VA equations with these cases. To this regard, we consider the 1D GPE with a high order nonlinearity of the type $\gamma|\psi|^{\alpha} \psi$ with $\alpha=2,4,6$

$$
i \psi_{t}+\psi_{x x}+\gamma|\psi|^{\alpha} \psi+\varepsilon \cos (2 x) \psi=0 .
$$

Using the same approach as before, one can readily show that the effective lagrangian in this case is

$$
L_{e f f}=\frac{A^{2}}{2}\left(\frac{\pi}{a}\right)^{1 / 2}\left[\frac{1}{2} a-\mu-\varepsilon e^{-1 / a}-\frac{A^{\alpha} \gamma}{\left(\frac{\alpha+2}{2}\right)^{3 / 2}}\right],
$$


from which the following VA equations are derived

$$
\begin{aligned}
\mu & =\frac{a}{2}-\varepsilon e^{-1 / a}-\frac{\gamma N^{\alpha / 2}}{\left(\frac{\alpha+2}{2}\right)^{1 / 2}}\left(\frac{a}{\pi}\right)^{\alpha / 4}, \\
N^{\alpha / 2} & =\frac{2 a}{\alpha \gamma}\left(\frac{\alpha+2}{2}\right)^{3 / 2}\left(\frac{\pi}{a}\right)^{\alpha / 4}\left(1-\frac{2 \varepsilon}{a^{2}} e^{-1 / a}\right),
\end{aligned}
$$

here $\alpha=2,4,6$. We see that the case $\alpha=2$ coincides with the case $d=1$ considered above, and the case $\alpha=4$ with the quintic VA equations derived in 13 .

Notice that for $\varepsilon=0$ Eq. (13) admits exact solutions also for $\alpha=4,6$. For the septic case, indeed, we have, using $\psi=u e^{i m t}, m=-\mu>0$, that

$$
\psi=\left(\frac{4 m}{\gamma}\right)^{1 / 6} \operatorname{sech}^{1 / 3}(3 \sqrt{m} x),
$$

is an exact solution with a norm

$$
N=\frac{2^{1 / 3} \Gamma^{2}\left(\frac{1}{3}\right)}{3 \Gamma\left(\frac{2}{3}\right) \gamma^{1 / 3} m^{1 / 6}}
$$

where $\Gamma(x)$ is the gamma function. The Hamiltonian for this solution is equal to

$$
H=\int_{-\infty}^{\infty}\left(\left|\psi_{x}\right|^{2}-\frac{\gamma}{4}|\psi|^{8}\right) d x=0.44 \frac{m^{5 / 6}}{\gamma^{1 / 3}} .
$$

From the above VA equations for the $2 \mathrm{D}$ case, one can derive the value of $N_{c}$ for small values of $\varepsilon \neq 0$ as: $N_{c}=4 \pi(1-8 \varepsilon \exp (-2))$. For $\varepsilon=0.2$ we obtain $N_{c}=9.845$ which is in reasonable agreement with the value 10.8 obtained from numerical simulations of the $2 \mathrm{D}$ case. We remark that for small values of $\varepsilon$ the soliton is very extended in space and resembles a Bloch wave modulated with an envelope. In this case an effective mass approximation may be appropriate which allows to replace the GPE field equation with a nonlinear Schrödinger equation with effective mass $m^{*}$ and nonlinearity $\beta$. In the $2 \mathrm{D}$ case we have

$$
i u_{t}+\alpha\left(u_{x x}+u_{y y}\right)+\beta|u|^{2} u=0
$$

where $\alpha=m^{*} / m, m^{*(-1)}=\left(\partial^{2} E / \partial k^{2}\right)_{k=0}$ and $\beta=\left(2 \pi / L^{2}\right) \int d^{2} r\left|\phi_{1,0}\right|^{4}$ (a similar equation can be written also for the $3 \mathrm{D}$ case) . In this approximation the norm of the gap Townes soliton can be evaluated as $N=\frac{N_{T}}{\alpha \beta}$. For deep optical lattices $(\varepsilon>5) \alpha$ can be approximated as $\alpha \approx \varepsilon^{1 / 4}$ and the norm $N \approx \frac{N_{T}}{\varepsilon^{1 / 4} \beta}$.

\section{Numerical study of gap-Townes solitons and delocalizing transitions}

In this section we investigate the existence curve of gap-Townes solitons in the $(N, \varepsilon)$ plane. As mentioned before, this curve coincides with the delocalizing 
transition curve which separates stable localized solutions from decaying ones. Its knowledge is therefore important for experimental investigations of multidimensional solitons. To this regard we remark that for the observation of multidimensional BEC solitons parameters should be chosen between the delocalizing and the collapsing curves. In the following we investigate the delocalizing curve by means of direct numerical integrations of the 2D and 3D GPE with periodic potential and by the corresponding 1D NLS systems with quintic and septic nonlinearities discussed above, respectively. The existence of gap-Townes solitons is then shown by direct numerical simulations of the multidimensional GPE using as initial conditions the above mentioned product states, which are found by solving the 1D GPE with higher nonlinearities by means of the self-consistent

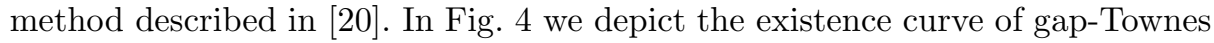
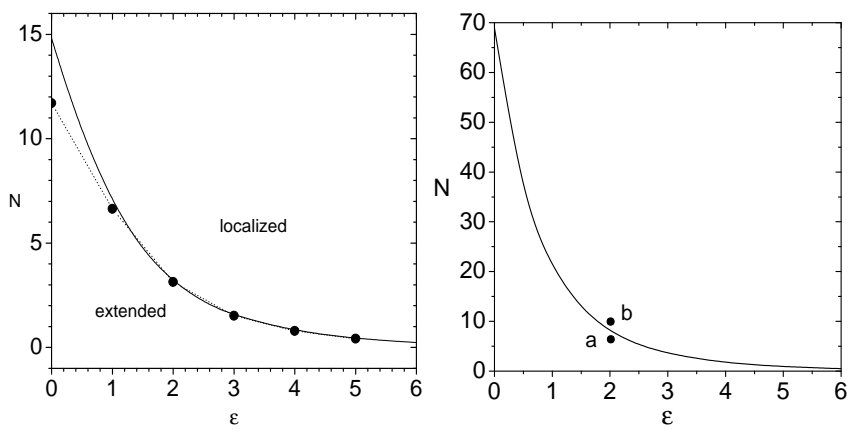

Fig. 4. Left panel: Existence curve of gap-Townes solitons of the 2D GPE for $\gamma=1$. The continuous line represents the delocalizing curve as obtained from the 1D quintic NLS approximation. The dots joined by thin dotted line represent numerical results obtained from direct integrations of the 2D GPE. Right panel: Existence curve of gap-Townes solitons of the 3D GPE for $\gamma=1$. The line represents the delocalizing curve as obtained from the 1D quintic NLS approximation, while the dots represent numerical results obtained from direct integrations of the 3D GPE.

solitons of the 2D GPE for $\gamma=1$ as obtained from numerical integrations of the 2D GPE. The corresponding curve obtained from the 1D quintic NLS approximation by means of a self-consistent approach is also shown. We see that for $\varepsilon>1$ the $1 \mathrm{D}$ quintic NLS curve agrees very well with that of the 2D GPE, the deviations becoming evident only for strengths of the OL which are less than $\varepsilon \approx 1$ (one recoil energy). This fact can be easily understood from the observation that for a fixed value of $\varepsilon$ there is one value of $N$ for which the gap Townes soliton exists and that by increasing $\varepsilon$ the corresponding value of $N$ decreases. This implies that for gap-Townes solitons in a strong OL the nonlinear interaction is effectively small, due to the potential barriers which prevent tunneling of matter into adjacent wells. On the contrary, in a shallow optical lattice the 
matter can easily tunnel through the barriers and the effective (attractive) nonlinearity can be larger. Since the separability ansatz used to link the 2D GPE to the 1D quintic NLS works well when the nonlinearity is small, it is clear that a discrepancy can arise at small values of $\varepsilon$. The fact that the quintic NLS equation deviates from the 2D GPE only for $\varepsilon<1$, however, makes the mapping between these two equations very convenient for practical calculations. A similar situa-

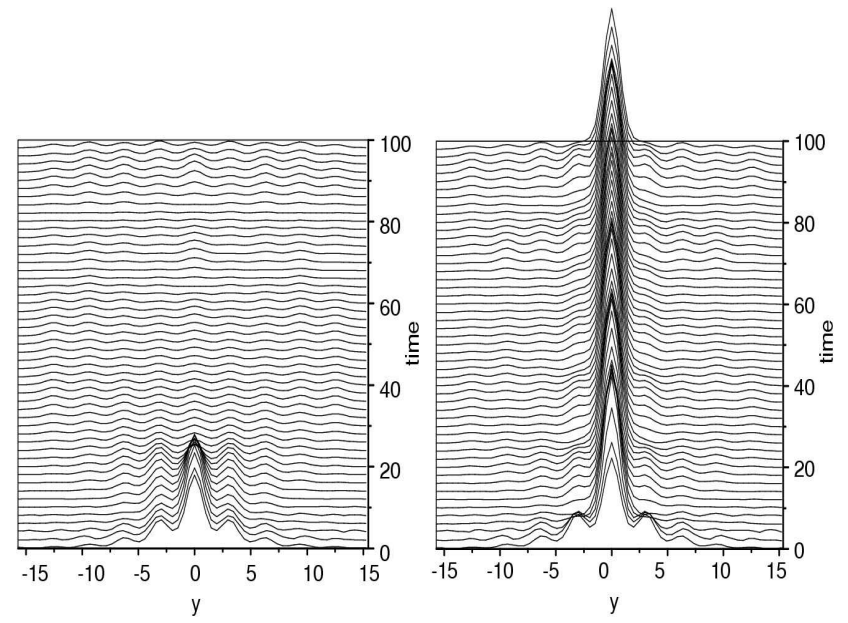

Fig. 5. Time evolution of the y-section of a 3D gap-Townes soliton obtained for $\varepsilon=2$ with slightly undercritical (left panel) and overcritical (right panel) number of atoms, corresponding to points a and b in Fig 4, respectively.

tion seems to be true also for the 3D case. In the right panel of Fig. 团we depict the existence curve of gap-Townes solitons of the 3D GPE for $\gamma=1$ as obtained from the 1D septic NLS approximation by means of a self-consistent approach. Due to the long computational times required in 3D simulations we have presented verification for only few points of the curve. A more complete analysis will require further investigation. In particular, the prediction of our analysis are checked by means of numerical integrations of the 3D GPE in correspondence of the two filled dots depicted in the right panel of Fig. 4 at $\varepsilon=2$, just above and below the delocalizing curve obtained from the septic 1D NLS equation. In Fig. 5 we show the time evolution of the y-section of a 3D gap-Townes soliton obtained for $\varepsilon=2$ in correspondence of these slightly undercritical (left panel) and overcritical (right panel) points. We see that, in analogy to what we observed in $2 \mathrm{D}$ case, the initial condition with an undercritical number of atoms leads to the complete delocalization of the state, while in the overcritical case a gap soliton state which remains stable over long time is formed. It is appropriate to mention that the exact profile of the 3D gap-Townes soliton, which separates these two behaviors is more difficult to obtain with the separability ansatz than for the 
previous $2 \mathrm{D}$ case, since in the $3 \mathrm{D}$ case this ansatz appears to be less accurate. The signature of the gap-Townes soliton state, however, is very clear as one can see from the early stages of the time evolution depicted in Fig. [5 (notice from the left panel that the undercritical state remains stable up to a time $t=20$ before starting to decay). This behavior strongly suggests the existence of gap-Townes solitons also in the 3D case.

We now address to another important property of the gap-Townes soliton which shows up in the transition to a gap soliton state for overcritical number of atoms. As it was already mentioned, even a slightly overcritical norm gives rise to a rapid shrinking of the gap-Townes soliton. Although at initial stage of evolution this behavior is similar to the collapse of ordinary Townes soliton, the final state is different. Specifically, gap-Townes soliton approaches the (final) gap state via long-lasting oscillations, as shown in Fig. 6. Each "reflection" from the broad state is accompanied by emission of linear waves, which can be viewed as a tunneling of matter from the localized mode into the extended one. This process also contributes to the damping of the oscillations. We remark that these oscillations, having a very regular behavior, could be used to detect the existence of gap-Town solitons in a real experiment.

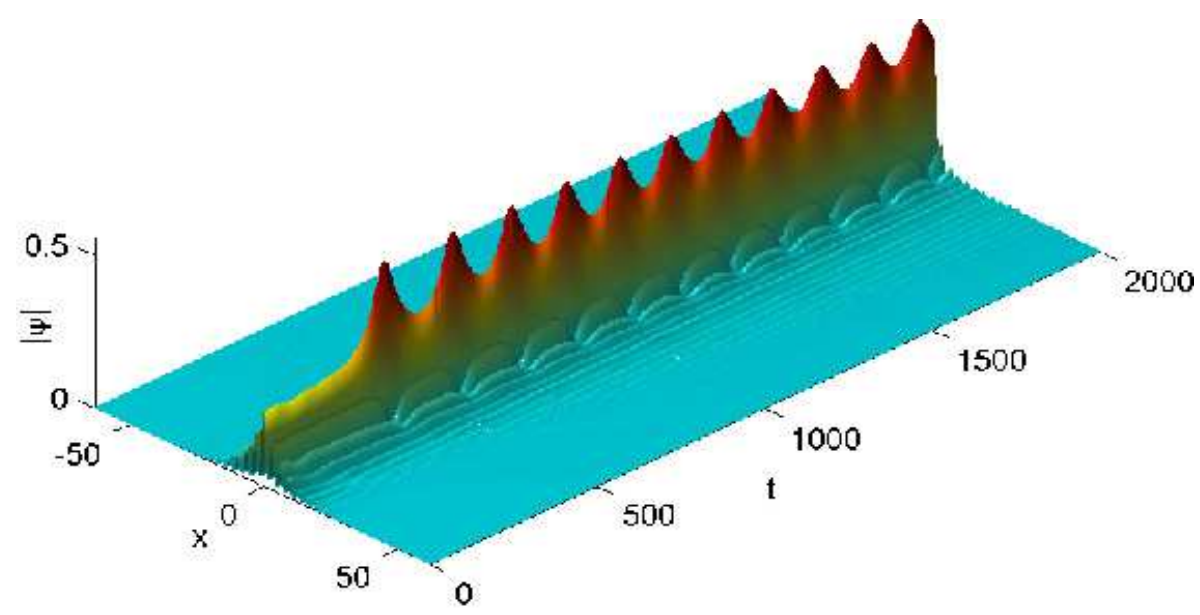

Fig. 6. Oscillations of the gap-Townes soliton when it collapses into the final gap state, according to Eq. (11) with initial state taken as the product solution constructed from two quintic NLS with a total norm increased by one percent. Shown is the $y=0$ cross section of the $2 \mathrm{D}$ profile.

\section{Conclusions}

In this paper we have shown the existence of gap-Townes solitons in the multidimensional (2D and 3D) Gross-Pitaevskii equation with a periodic potential and 
discussed its link with the phenomenon of delocalizing transition. These solutions have the peculiarity of being unstable under small fluctuations of the number of atoms and separate localized (soliton like) states from extended (Bloch like) ones. The existence curve in the parameter space of this particular solution is very useful since it provides the lower threshold for the existence of localized states. The gap-Townes solitons discussed in this paper are a natural generalization of the Townes solitons of the nonlinear Schrödinger equation (without periodic potential). The existence of Townes solitons in a nonlinear glass sample modelled by the NLS equation was experimentally demonstrated in Ref. 21. The fact that the transition from a gap-Townes soliton to a gap soliton is always accompanied by regular oscillations gives the possibility to indirectly observe the multidimensional gap-Townes solitons discussed in this paper in real experiments. In particular we expect these solitons to be observed both in multidimensional BECs in OL and in nonlinear optics systems, including 2D and 3D photonic crystals and arrays of nonlinear optical waveguides.

\section{Acknowledgements}

MS acknowledge partial financial support from a MURST-PRIN-2003 and a MIUR-PRIN-2005 initiatives. FKhA and BBB wish to thank the Department of Physics "E. R. Caianiello" for hospitality and the University of Salerno for financial support.

\section{References}

1. S. Burger, K. Bongs, S. Dettmer, W. Ertmer, K. Sengstock, A. Sanpera, G. V. Shlyapnikov, and M. Lewenstein, Phys. Rev. Lett. 83, 5198 (1999).

2. L. Khaykovich, F. Schreck, G. Ferrari, T. Bourdel, J. Cubizolles, L. D. Carr, Y. Castin and C. Salomon, Science 296, 1290 (2002).

3. K. E. Strecker, G. B. Partridge, A. G. Truscott and R. G. Hulet, Nature 417, 150 (2002).

4. B. Eiermann, P. Treutlein, Th. Anker, M. Albiez, M. Taglieber, K.-P. Marzlin, and M. K. Oberthaler, Phys. Rev. Lett. 91, 060402 (2003).

5. C. Sulem and P. L. Sulem, The Nonlinear Schrödinger Equation: Self-focusing and Wave Collapse, Springer, 1999.

6. R. Y. Chiao, E. Garmire and C. H. Townes, Phys. Rev. Lett. 13, 479 (1964).

7. B. B. Baizakov, V. V. Konotop, and M. Salerno, J. Phys. B: At. Mol. Opt. Phys., 35,5105 (2002).

8. N. K. Efremidis, S. Sears, D. N. Christodoulides, J. W. Fleischer and M. Segev, Phys. Rev. E 66, 046602 (2002).

9. E. Ostrovskaya and Yu.S. Kivshar, Phys. Rev. Lett. 90, 160407 (2003).

10. B. B. Baizakov, B. A. Malomed, and M. Salerno, Eurphys. Lett. 63, 642 (2003).

11. D. Mihalache, D. Mazilu, F. Lederer, B. A. Malomed, L.-C. Crasovan, Y. V. Kartashov, and L. Torner, Phys. Rev. A 72, 021601(R) (2005).

12. B. B. Baizakov and M. Salerno, Phys.Rev. A 69, 013602 (2004).

13. F. Kh. Abdullaev and M. Salerno, Phys. Rev. A 72, 033617 (2005). 
14 Mario Salerno ${ }^{1}$, F. Kh. Abdullaev ${ }^{2}$, and B. B. Baizakov ${ }^{2}$

14. G.L. Alfimov, V.V. Konotop, P. Pacciani, Phys. Rev. A 75, 023624 (2007).

15. H.A. Cruz, V.A. Brazhnyi, V.V. Konotop, and M. Salerno, Physica D (2008), doi:10.1016/j.physd.2008.09.008.

16. L. Berge, Phys. Rep. 303, 259 (1998).

17. N. K. Efremidis, J. Hudock, D. N. Christodoulides, J. W. Fleischer, O. Cohen, and M. Segev, Phys. Rev. Lett. 91, 213906 (2003).

18. N. G. Vakhitov, A. A. Kolokolov, Radiophysics and Quantum Electronics, 16, 783 (1973).

19. N. Akhmediev, M. P. Das and A. V. Vagov, Int. J. Mod. Phys. B 13625 (1999).

20. Mario Salerno, Laser Physics 15, No.4, 620 (2005).

21. K. D. Moll, A. L. Gaeta, G. Fibich, Phys. Rev. Lett. 90, 203902 (2003). 